\title{
The Effect of the Auxiliary Electrode on the Microplasma Generated in a Plasma Display With a Coplanar Gap
}

\author{
Seung Hun Kim, Jeong Hun Mun, Kyung Cheol Choi, Member, IEEE, and Seong-Eui Lee
}

\begin{abstract}
The microplasma modes generated in a plasma display with a coplanar gap and an auxiliary electrode were investigated using the discharge current peak time (discharge delay) and discharge current. Three types of modes were classified as follows: Mode 1) showed a decrease in both the discharge current and the discharge delay, Mode 2) showed a decrease in the discharge current and an increase in the discharge delay, and Mode 3) showed an increase in the discharge current and a decrease in the discharge delay. The infrared efficiency increased in Modes 1) and 2) with an increase in the auxiliary pulse voltage. However, in Mode 3), the IR efficiency started to decrease. According to the measurement results, Modes 1) and 2) are suitable for microplasma generated in a coplanar gap with an auxiliary electrode to obtain high efficacy.
\end{abstract}

Index Terms-Auxiliary electrode, coplanar gap, discharge current peak time, discharge delay, infrared (IR) efficiency, IR peak time, microplasma, plasma display.

\section{INTRODUCTION}

$\mathbf{M}$ ICROPLASMA, often referred to as microdischarge, is defined as a spatially confining plasma having a dimension of less than $1 \mathrm{~mm}$ and operating pressures in the order of several hundred torr. Microplasma generates stable glow discharges in a wide variety of gases and is capable of significant shifts in both temperature and energy distribution. These characteristics allow for applications in medical devices, display, environmental equipment, ultraviolet (UV) radiation sources, plasma reactors, and plasma cathodes; many types of which were developed in the past decade [1]. Recently, microplasma has been utilized in display applications such as alternatingcurrent plasma display panels (ac-PDPs) and thin-film transistor liquid-crystal display (TFT-LCD) back light unit (BLU). AC-PDPs have two essential advantages for large-size displays compared to a cathode ray tube and a field emission display. The first is that ac-PDPs do not need thick glass plates due to the nearly identical pressure inside and outside the display. The second advantage is that the driving of the large-size display is easy due to the small capacitance between the electrodes [2], [3]. These advantages have led to good performance for ac-

Manuscript received November 18, 2006; revised February 8, 2007. This work was supported by the Basic Research Program, Korea Science and Engineering Foundation under Grant R01-2006-000-10075-0.

S. H. Kim, J. H. Mun, and K. C. Choi are with the Department of Electrical Engineering and Computer Science, Korea Advanced Institute of Science and Technology, Daejeon 305-701, Korea (e-mail: kyungcc@ee.kaist.ac.kr).

S.-E. Lee is with the Department of Advanced Materials Engineering, Korea Polytechnic University, Shihung, Korea (e-mail: selee@kpu.ac.kr).

Color versions of one or more of the figures in this paper are available online at http://ieeexplore.ieee.org.

Digital Object Identifier 10.1109/TPS.2007.895225
PDPs and have allowed them to enter the consumer electronics market. However, the luminous efficacy of ac-PDPs is typically lower than that of other displays due to the inefficiency of the glow discharges. Only $14 \%$ of the input power is used for the emission of UV photons, a characteristic that becomes problematic for large-size ac-PDPs [4]. Therefore, improvement in the luminous efficacy is now a crucial issue in the current acPDP field. Many researchers have reported various methods such as various coplanar-gap discharges [5], high-Xe-content discharges [6], [7], and a new driving waveform [8] to achieve a high luminous efficacy; mostly, these have showed good results. In contrast, the actual phenomena related to microplasma in ac-PDPs are not yet completely understood due to its exceedingly small discharge cell. For this reason, understanding the characteristics of microplasma in ac-PDPs remains a challenge. There have been several reports that have made progress toward clarifying the microplasma phenomena in ac-PDPs [9]-[11], but only a thorough understanding of these characteristics will allow the achievement of high luminous efficacy in ac-PDPs.

In previous reports, the new structure with an auxiliary electrode in the coplanar gap was proposed [12], [13]; these showed some improvement of the luminous efficacy in an ac-PDP. The auxiliary electrode affected the metastable and excited particles through a weak excitation and played a role in reducing discharge current, which improved luminous efficacy. However, the effect of the auxiliary electrode on the microplasma itself remains unknown.

Therefore, this paper investigates microplasma modes in accordance with an auxiliary electrode in a plasma display with a coplanar gap and a gap distance, including the discharge current peak time, discharge current, infrared (IR) initial time, and IR efficiency. From the results, the effect of the auxiliary electrode on differing microplasma modes is characterized.

\section{Test PAnel Preparation}

A schematic diagram of the test panel with a coplanar gap and an auxiliary electrode is shown in Fig. 1. On the front plate, the sustain and auxiliary electrodes were made of conductive paste and patterned via a photolithography method. The width of the electrodes and the distance between the sustain electrodes were 100 and $500 \mu \mathrm{m}$, respectively. To investigate the effects of the position of the auxiliary electrode, the distance between the $X$ - and the auxiliary electrode, defined as the gap distance $d$, was varied from 50 to $200 \mu \mathrm{m}$. Following this, the electrodes were coated with a 30- $\mu$ m-thick transparent dielectric layer using a screen-print method. 




Fig. 1. Schematic diagram of the test panel with coplanar and the auxiliary electrodes.

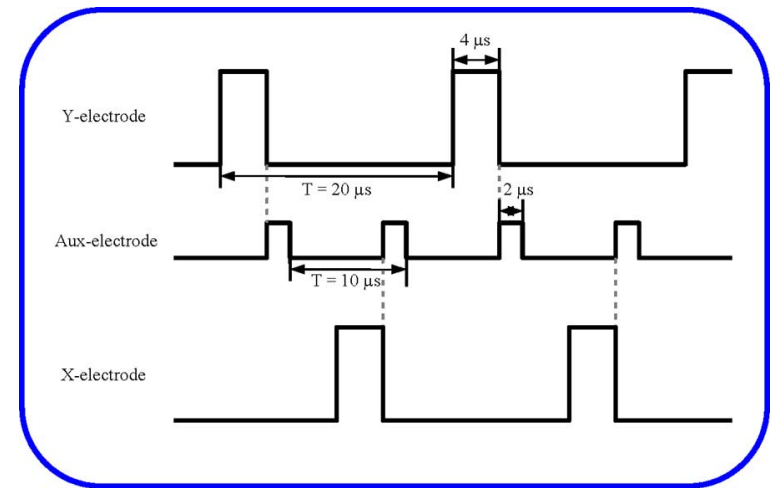

Fig. 2. Pulse waveforms applied to the sustain and the auxiliary electrodes.

On the rear plate, a $40-\mu \mathrm{m}$-thick dielectric layer, barrier ribs, and a green phosphor layer were formed via a screen-printing method. The barrier rib height was $100 \mu \mathrm{m}$. A Ne $+4 \%$ Xe gas mixture was used as a discharge gas, with a total gas pressure of 500 torr.

The pulse waveforms applied to the sustain and auxiliary electrodes are shown in Fig. 2. The frequency of sustain pulse was $50 \mathrm{kHz}$ with a pulse width of $4 \mu \mathrm{s}$. The frequency of the auxiliary pulse was $100 \mathrm{kHz}$ with a pulse width of $2 \mu \mathrm{s}$, which was applied immediately after the sustain pulse. The sustain voltage was higher compared to that used for the conventional ac-PDP; as the protecting layer, $\mathrm{MgO}$, was not used in this paper. The sustain voltage was in the range of $400-600 \mathrm{~V}$. The auxiliary voltage varied from 0 to $200 \mathrm{~V}$.

\section{RESUlTS AND DISCUSSION}

The discharge current peak time and discharge current in a cell were measured to investigate the effects of the position of the auxiliary electrode. The discharge current peak time is defined as the time which takes the discharge current peak value. Fig. 3 shows the discharge current peak time and discharge current obtained from the coplanar-gap discharges as a function of the voltage of the pulse applied to the auxiliary electrode in accordance with the gap distance $d$, as shown in Fig. 1 .

As shown in Fig. 3, the discharge current peak time and discharge current showed different tendencies as the auxiliary pulse voltage increased. When the gap distance $d$ was $50 \mu \mathrm{m}$, the discharge current peak time decreased until the auxiliary pulse voltage was $60 \mathrm{~V}$, thereafter, increased in the range of the auxiliary pulse voltage of $60-110 \mathrm{~V}$, and decreased again when the auxiliary pulse voltage was over $110 \mathrm{~V}$. When the gap distance $d$ was $100 \mu \mathrm{m}$, the discharge current peak time increased as the auxiliary pulse voltage increased up to $90 \mathrm{~V}$ and decreased as the auxiliary pulse voltage increased. The discharge current peak time with a gap distance of 150 and $200 \mu \mathrm{m}$ increased from the start point of the grounded auxiliary electrode to around $50 \mathrm{~V}$ as the discharge current decreased, and thereafter decreased as the discharge current increased. The IR emission initial time was also measured to investigate the effects of the position of the auxiliary electrode. The IR emission initial time is defined as the time which initiates the IR emission. The IR emission initial time is generally identical to the discharge delay time [14]; the results were identical to those of the discharge current peak time. In addition, the discharge current decreased with a low voltage of the auxiliary pulse and increased with a high voltage of this pulse. In the case of $d=50,100$, and $150 \mu \mathrm{m}$, the discharge current reached its minimum point at an auxiliary pulse voltage of around $100 \mathrm{~V}$. In the case of $d=200 \mu \mathrm{m}$, the discharge current has its minimum value at an auxiliary pulse voltage of $80 \mathrm{~V}$.

On the basis of the measurements of the discharge current peak time and discharge current, the microplasma generated in the coplanar-gap electrode with the auxiliary electrode were classified as follows:

Mode 1) decrease in both the discharge current and the discharge delay;

Mode 2) decrease in the discharge current and increase in the discharge delay;

Mode 3) increase in the discharge current and decrease in the discharge delay.

To illustrate the phenomena of the three microplasma modes generated in a coplanar gap with the auxiliary electrode, discharge models were suggested, as shown in Fig. 4. The left hand-side panel structure on the right in the figure shows the wall-charge state after the sustain pulse is applied to the $Y$-electrode, and right hand-side panel structure on the right in the figure shows the wall-charge state after the pulse is applied to the auxiliary electrode.

In the case of Mode 1), the negative charges are accumulated on the $Y$-electrode, and the positive charges are accumulated on both the $X$ - and auxiliary electrodes, as shown in Fig. 4(a). When the auxiliary pulse is applied during the after glow, there is no discharge between the $Y$ - and auxiliary electrodes, as the electric field is not strong enough to cause a discharge due to low voltage of the auxiliary pulse. However, a small amount of positive charge on the auxiliary electrode will recombine with the negative charge on the $Y$-electrode. At that time, this reaction creates excitation and metastable particles, which are used as the priming particles in the next periodical sustain discharge. Consequently, the discharge current decreases due to the reduced wall charges, which are due to the auxiliary pulse. Additionally, the discharge delay decreases as a result of the prime particles generated from the reaction between the negative and positive wall charges. It is also possible for the 


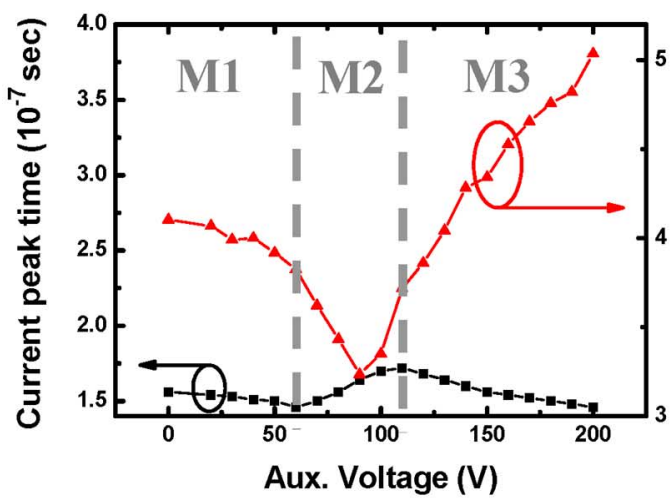

(a)

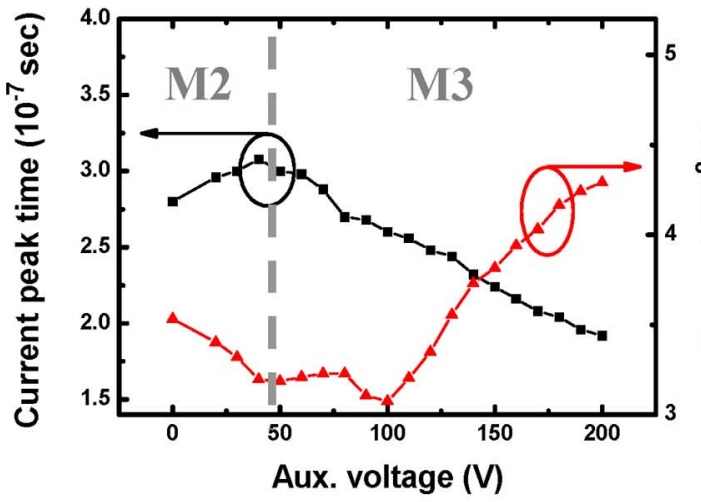

(c)

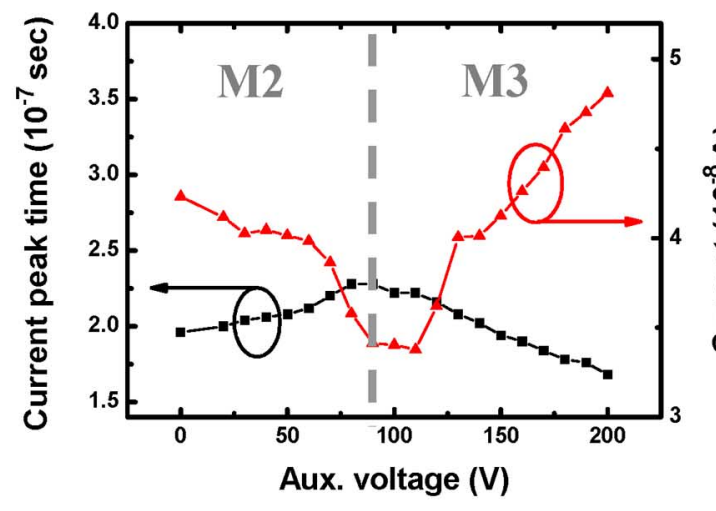

(b)

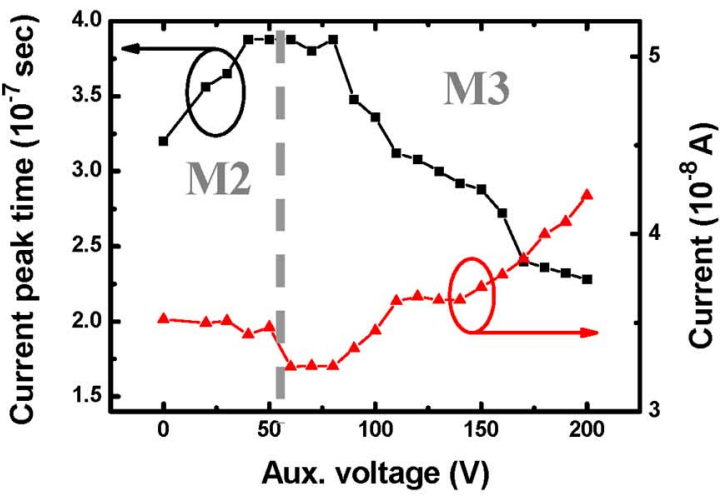

(d)

Fig. 3. Current peak time and discharge current as a function of the voltage of the pulse applied to the auxiliary electrode in accordance with the gap distance $d$ (a) $d=50 \mu \mathrm{m}$. (b) $d=100 \mu \mathrm{m}$. (c) $d=150 \mu \mathrm{m}$. (d) $d=200 \mu \mathrm{m}$.

discharge delay to remain the same, as the effect of the prime particles can be offset by the reduced wall charges.

In the case of Mode 2), the gap distance $d$ is shorter, and voltage of the auxiliary pulse is higher compared to those of Mode 1). When the pulse is applied to the auxiliary electrode, there should be a weak discharge between the $Y$ - and the auxiliary electrode as the electric field is strong enough to create a discharge due to the smaller gap distance and higher voltage. After applying the auxiliary pulse, more wall charges are erased compared to what occurs in Mode 1). Consequently, the reduced wall charge leads to a decreased discharge current and increased discharge delay.

In the case of Mode 3), the auxiliary pulse voltage is much higher compared to this voltage level in both Modes 1) and 2). When the pulse is applied to the auxiliary electrode, there is a strong discharge between the $Y$ - and the auxiliary electrode, as the electric field is sufficiently high. This strong discharge converts the state of the wall charges on the auxiliary electrode to the opposite polarity. Consequently, the next periodical sustain discharge mainly occurs between the $X$ - and the auxiliary electrode rather than between the $X$ - and $Y$-electrodes because the discharge path between the $X$ - and the auxiliary electrode is shorter than that between the $X$ - and $Y$-electrodes.

According to the above mode analysis, a gap distance of 50 was seen in Modes 1), 2), and 3), while gap distances of 100,
150, and $200 \mu \mathrm{m}$ were seen in Modes 2) and 3), as shown in Fig. 3. As the gap distance increased, Mode 1) disappeared; as the electric field between the $Y$ - and the auxiliary electrode increased, it led to a discharge. Therefore, a critical distance for a mode transition may exist in the distance between the gap distances of 50 and $100 \mu \mathrm{m}$. Additionally, the growth rate of the discharge current decreased according to the increase of the gap distance in Mode 3). As mentioned previously, the discharges between the $X$ - and the auxiliary electrode or the $Y$ - and the auxiliary electrode were dominant in Mode 3). Therefore, a smaller gap distance had a larger electric field, which caused the high growth rate of the discharge current.

Fig. 5 shows the discharge-current decay and sustain transition voltages as a function of the gap distance and the stable operation region in accordance with the sustain transition voltage. The discharge-current decay is defined as the dischargecurrent difference between the discharge current in a floated auxiliary electrode and the discharge current in a grounded auxiliary electrode. The sustain transition voltage is defined as the voltage which maintains microplasma to be in an ON-state when a pulse is applied to the auxiliary electrode.

As shown in Fig. 5(a), the discharge-current decay was increased as the gap distance increased. As mentioned previously, the higher electric field from the reduced distance between the $Y$ - and the auxiliary electrode results in the fact that more 
(a)
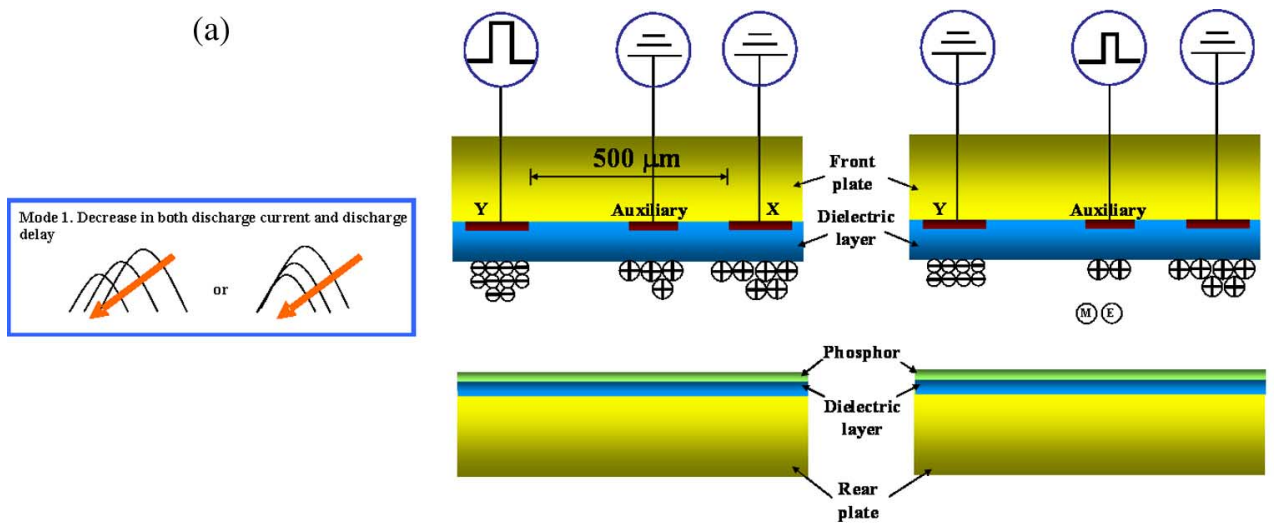

(b)
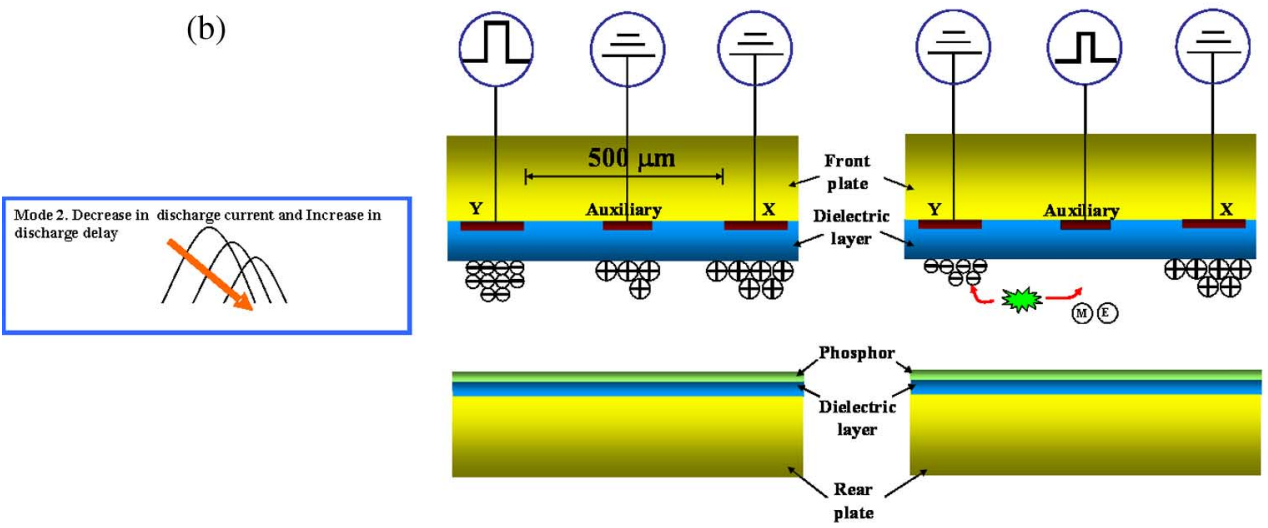

(c)
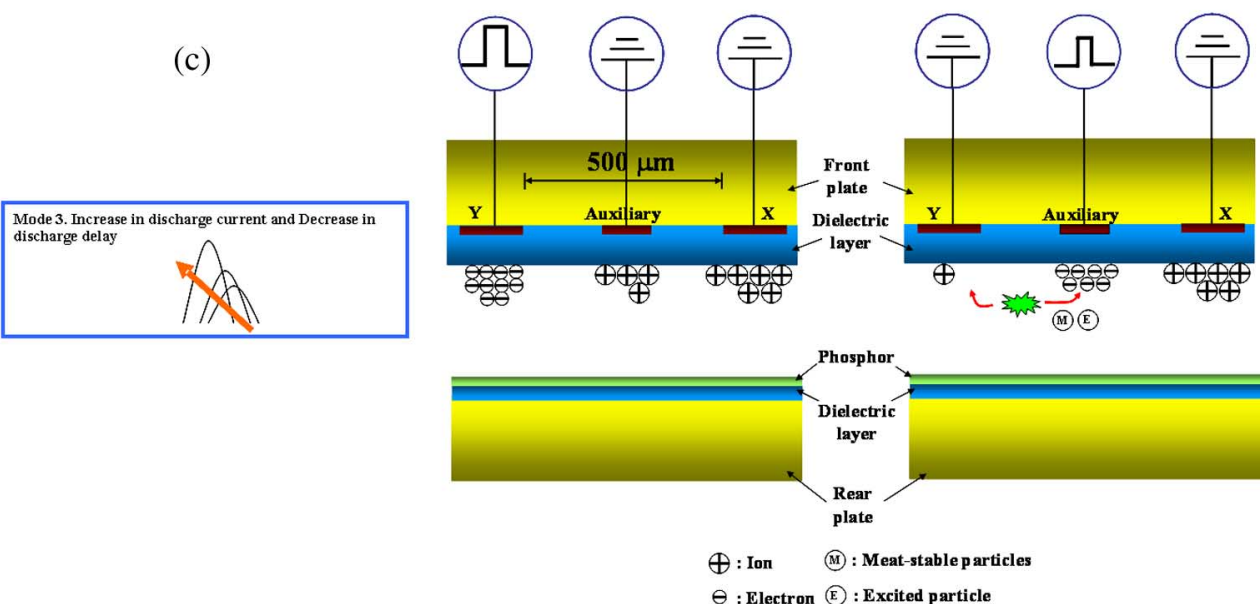

Fig. 4. Discharge models illustrating the three microplasma modes. (a) Model of Mode 1). (b) Model of Mode 2). (c) Model of Mode 3).

wall charges were erased. Therefore, the discharge current was more decreased, as shown in Fig. 5(a). Fig. 5(a) also shows the sustain transition voltage as a function of the gap distance. The sustain transition voltage decreased as the gap distance increased. As mentioned previously, the discharges between the $X$ - and the auxiliary electrode or the $Y$ - and the auxiliary electrode were dominant in Mode 3). The microplasma turned off at the long distance between the $Y$ - and the auxiliary electrode, as the electric field was not strong enough to create a discharge between the $Y$ - and the auxiliary electrode. Therefore, the sustain transition voltage decreased as the gap distance increased.

The IR spectra of 823 and $828 \mathrm{~nm}$ were measured to investigate the vacuum ultraviolet (VUV) efficiency at second hand. It is generally known that the VUV emission intensities of 147 and $173 \mathrm{~nm}$ are closely related and proportional to the IR emission intensities of 823 and $828 \mathrm{~nm}$ [15]. Fig. 6 shows the IR efficiency as a function of the auxiliary pulse voltage in accordance with the gap distance.

The IR efficiency increased as the gap distance increased up to $150 \mu \mathrm{m}$. The decay rate of the discharge current increased as the gap distance increased. Therefore, the IR efficiency increased until the gap distance became $150 \mu \mathrm{m}$. However, the IR efficiency in the case of a gap distance of $200 \mu \mathrm{m}$ had a different result. The efficiency in the case of a gap distance of $200 \mu \mathrm{m}$ was slightly lower in the range of a low auxiliary pulse voltage and higher in range of a high auxiliary pulse voltage compared to that at $150 \mu \mathrm{m}$ because the decay rate of the discharge current with a gap distance of $200 \mu \mathrm{m}$ was lower in the range of a low auxiliary pulse voltage, and the growth rate of 


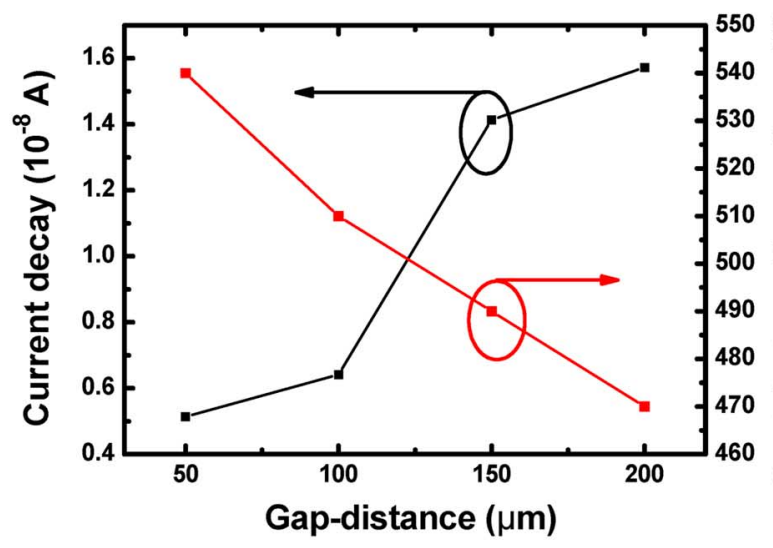

(a)

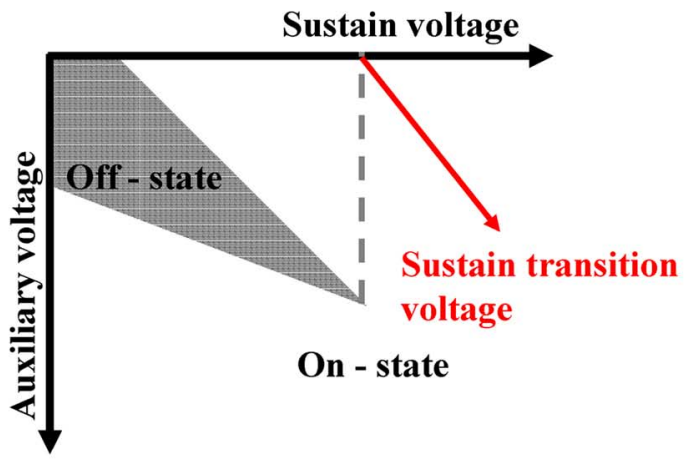

(b)

Fig. 5. (a) Discharge-current decay and the sustain transition voltage as a function of gap distance. (b) Stable operation region in accordance with the sustain transition voltage.



Fig. 6. IR efficiency as a function of the auxiliary pulse voltage in accordance with the gap distance.

the discharge current was lower in the range of a high auxiliary pulse voltage. The three microplasma modes are also shown in Fig. 6. In Mode 1), the IR efficiency increased. However, this mode can be only shown in the case of a gap distance of $50 \mu \mathrm{m}$. In Mode 2), the IR efficiency increased as the auxiliary pulse voltage increased. In Mode 3), there were two types of regions: a region for an increase in the IR efficiency and a region for a decrease in the IR efficiency. The IR efficiency of the discharge in the case of gap distances of 100 and $150 \mu \mathrm{m}$ increased until the auxiliary pulse voltage became $100 \mathrm{~V}$; thereafter, it decreased. The IR efficiency of the discharge in the case of a gap distance of $200 \mu \mathrm{m}$ increased until the auxiliary pulse voltage became $140 \mathrm{~V}$; thereafter, it decreased.

\section{CONCLUSION}

The discharge current peak time, IR initial time, discharge current, and IR efficiency of a plasma display with a coplanar gap and a gap distance were measured to investigate microplasma modes in accordance with the effect of the auxiliary electrode. According to measurement results of the discharge current peak time and discharge delay, the microplasma modes generated in the coplanar gap with the auxiliary electrode were classified as follows: Mode 1) showed a decrease in both the discharge current and the discharge delay, Mode 2) showed a decrease in the discharge current and an increase in the discharge delay, and Mode 3) showed an increase in the discharge current and a decrease in the discharge delay. From these three plasma modes, microplasma with a gap distance of $50 \mu \mathrm{m}$ is applied in Modes 1), 2), and 3), and microplasma with gap distances of 100,150 , and $200 \mu \mathrm{m}$ are applied for 
Modes 2) and 3) only. As the gap distance and electric field increased, Mode 1) disappeared. Therefore, a critical distance of a mode transition in a gap distance between 50 and $100 \mu \mathrm{m}$ may exist. Additionally, the growth rate of the discharge current decreased in accordance with an increase in the gap distance in Mode 3), as a smaller gap distance had a larger electric field, which caused a higher growth rate of the discharge current. The discharge-current decay increased, and the sustain transition voltage decreased as the gap distance increased. Finally, the IR efficiency increased in Modes 1) and 2) as the auxiliary pulse voltage increased, as the discharge current decreased. However, in Mode 3), the IR efficiency started to decrease due to the increase in the discharge current. From these results, it was found that microplasma in Modes 1) and 2) is useful for obtaining high efficacy in a plasma display with a coplanar gap and an auxiliary electrode.

\section{REFERENCES}

[1] K. H. Becker, K. H. Schoenbach, and J. Eden, "Microplasmas and applications," J. Phys. D: Appl. Phys., vol. 39, no. 3, pp. R55-R70, Feb. 2006.

[2] H. Uchiike and T. Hirakawa, "Color plasma displays," Proc. IEEE, vol. 90, no. 4, pp. 533-539, Apr. 2002.

[3] J. P. Boeuf, "Plasma display panels: Physics, recent developments and key issues," J. Phys. D: Appl. Phys., vol. 36, no. 6, pp. R53-R79, Mar. 2003.

[4] J. S. Seo, W. J. Chung, C. K. Yoon, J. K. Kim, and K. W. Whang, "Discharges efficiency analysis of an ac PDP by numerical simulation," in Proc. 6th Int. Display Workshop, 1999, pp. 667-670.

[5] K. C. Choi, N. H. Shin, K. S. Lee, J. Shin, and S. E. Lee, "Study of various coplanar gaps discharges in ac plasma display panel," IEEE Trans. Plasma Sci., vol. 34, no. 2, pp. 385-389, Apr. 2006.

[6] G. Oversluizen, S. de Zwart, M. F. Gillies, T. Dekker, and T. J. Vink, "The route toward a high efficacy PDP: Influence of Xe partial pressure, protecting layer, and phosphor saturation," Microelectron. J., vol. 35, no. 4, pp. 319-324, Apr. 2004.

[7] W. J. Chung, B. J. Shin, T. J. Kim, H. S. Bae, J. H. Seo, and K. W. Whang, "Mechanism of high luminous efficient discharge with high pressure and high Xe content in ac PDP," IEEE Trans. Plasma Sci., vol. 31, no. 5, pp. 1038-1043, Oct. 2003.

[8] B. G. Cho, H. S. Tae, K. Ito, J. W. Song, E. Y. Jung, J. C. Ahn, and N. S. Jung, "A new driving waveform for improving luminous efficiency in ac PDP with large sustain gap under high Xe content," IEEE Trans. Plasma Sci., vol. 34, no. 2, pp. 390-396, Apr. 2006.

[9] K. C. Choi, B. J. Rhee, and H. N. Lee, "Characteristics of charged and metastable species in micro-discharge of ac plasma display panel," IEEE Trans. Plasma Sci., vol. 31, no. 3, pp. 329-332, Jun. 2003.

[10] K. W. Whang and J. K. Kim, "Diagnostics of the microdischarge in an alternating current plasma display panel (ac PDP)," IEEE Trans. Plasma Sci., vol. 34, no. 2, pp. 311-316, Apr. 2006.

[11] G. Oversluizen and T. Dekker, "Diagnostics of PDP micro-discharges," IEEE Trans. Plasma Sci., vol. 34, no. 3, pp. 305-310, Apr. 2006.

[12] K. Tachibana, S. Kawai, H. Asai, N. Kikuchi, and S. Sakamoto, "Characteristics of $\mathrm{Ne}-\mathrm{Xe}$ microplasma in unit discharge cell of plasma display panel equipped with counter sustain electrodes and auxiliary electrodes," J. Phys. D: Appl. Phys., vol. 38, no. 11, pp. 1739-1749, 2005.

[13] K. C. Choi, N. H. Shin, S. C. Song, J. H. Lee, and S. D. Park, "A new ac plasma display panel with auxiliary electrode for high luminous efficacy," IEEE Trans. Electron Devices, vol. 54, no. 2, pp. 210-218, Feb. 2007.

[14] K. C. Choi, S. H. Kim, B. J. Shin, J. W. Kang, K. Y. Choi, and E. H. Yoo, "Effects of $\mathrm{Kr}, \mathrm{N}_{2}$, and Ar on address discharge time lag in ac plasmadisplay panel with high xenon content," IEEE Trans. Electron Devices, vol. 53, no. 9, pp. 2410-2413, Sep. 2006.

[15] E. H. Choi, J. C. Ahn, M. W. Moon, Y. Jung, M. C. Choi, Y. H. Seo, G. S. Cho, H. C. Uhm, K. Tachibana, K. W. Whang, and M. Cristiansen, "Vacuum ultraviolet luminous efficiency and plasma ion density in alternating current plasma display panels," Appl. Phys. Lett., vol. 81, no. 18, pp. 3341-3343, Oct. 2002.

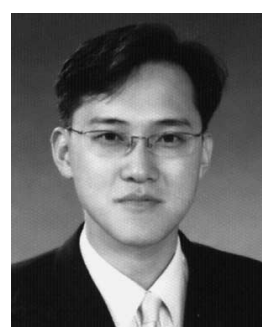

Seung Hun Kim received the B.S. degree from Chungnam National University, Daejeon, Korea, in 2003, and the M.S. degree in electrical engineering from the Korea Advanced Institute of Science and Technology, Daejeon, in 2005, where he is currently working toward the Ph.D. degree in electric engineering.

His research interests include plasma display panels and microplasma applications.

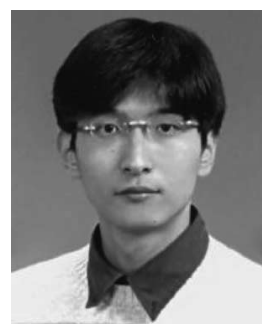

Jeong Hun Mun received the B.S. degree in electrical engineering from Kyungbook National University, Daegu, Korea, in 2006. He is currently working toward the M.S. degree in electric engineering at Korea Advanced Institute of Science and Technology, Daejeon, Korea.

His research interests include plasma display panels and microplasma applications.

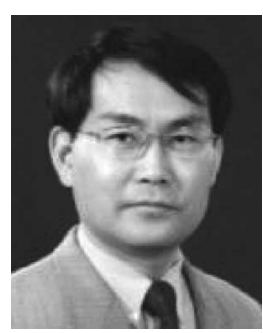

Kyung Cheol Choi (M'04) received the B.S. degree from the Department of Electrical Engineering, Seoul National University (SNU), Seoul, Korea, in 1986, and the M.S. and Ph.D. degrees in plasma engineering from SNU in 1988 and 1993, respectively.

He was with the Institute for Advanced Engineering, Seoul, from 1993 to 1995, where his work focused on the design of field emission display devices. He was a Research Scientist in the Microbridge Plasma Display Panel (PDP), Spectron Corporation of America, Summit, NJ, from 1995 to 1996. He was a Senior Research Scientist at Hyundai Plasma Display, Hawthorne, NY, from 1996 to 1998, where his work was to continue on developing plasma display technology. From 1998 to 1999, he was involved in the development of an ac 40-in PDP at Advanced Display R\&D Center, Hyundai Electronics Industries, Gyounggi-do, South Korea, as a Senior Research Scientist. From 2000 to 2004, he had been an Associate Professor in the Department of Electronics Engineering, Sejong University, Seoul. He also was in charge of the Information Display Research Center supported by Korean Ministry of Information and Communication. Since February 1, 2005, he has been an Associate Professor in the School of Electrical Engineering and Computer Science, Korea Advanced Institute of Science and Technology, Daejeon, Korea. His research interests include PDP, information displays, and microplasma applications. He is an Associate Editor of IEEE/OSA Journal of Display Technology.

Dr. Choi is a member of the Society for Information Display and the Korean Information Display Society.



Seong-Eui Lee received the B.S., M.S., and Ph.D. degrees in 1988, 1990, and 1995, respectively, from Seoul National University, Seoul, Korea. During his $\mathrm{Ph} . \mathrm{D}$. degree, he worked on the phase transformation kinetics of amorphous powder.

After his gradation, he was with Hyundai Electronics, Korea, and worked as a Senior Researcher at Hyundai Plasma Display Research Center, Japan, for 26-in XGA plasma display panel (PDP) project. After his PDP work, he was with iFire Technology Inc., as a Senior Researcher for the Thick Film Technology Development Group. After returning to Korea, he worked as a Principal Engineer at Samsung Advanced Institute of Technology for thick film related display development, particularly PDP and flexible display. He recently joined Department of Advanced Materials Engineering, Korea Polytechnic University, Shihung, Korea, as a Faculty Member. His main areas of interests are the process development and integration for display based on thick film or printing technology. He has published more than 20 papers in journal and conference proceedings and is the holder of more than 40 patents. 